\title{
T-Wave Alternans and Autonomic Nervous System Activity During Orthostatic Stress after 5 Days of Head-Down Bed-Rest
}

\author{
A. Martín-Yebra*, E.G. Caiani, V. Monasterio, A. Pellegrini, P. Laguna and J.P. Martínez
}

\begin{abstract}
Reports of ventricular arrhythmias during spaceflights raise the question of whether microgravity increases sudden cardiac death risk. We aimed at studying changes in T-wave alternans (TWA) together with Autonomic Nervous System activity during tilt-table (TT) testing before and after five days of $-6^{\circ}$ head-down bed-rest (HDBR), simulating exposure to microgravity. ECG signals were obtained in 22 males during TT, before and immediately after the end of HDBR, analyzed for multilead TWA detection and spectral analysis of heart rate variability. No differences in TWA indices before and after HDBR were found. However, unbalanced sympato-vagal response to TT already before HDBR together with higher TWA values were found in subjects with lower orthostatic tolerance time after HDBR.
\end{abstract}

\section{INTRODUCTION}

Microgravity leads to cardiovascular deconditioning, inducing significant changes in autonomic and cardiovascular systems, which may adversely influence cardiac repolarization with effects on cardiac rhythm disturbances [1], [2]. In this context T-wave alternans, (TWA), reflecting temporal and spatial repolarization heterogeneity and regarded as a noninvasive risk marker for predicting sudden cardiac death and ventricular vulneravility [3], could be affected. The head-down $\left(6^{\circ}\right)$ bed-rest (HDBR) model represents a way to induce and study the effects of exposure to simulated microgravity on the cardiovascular system.

We hypothesized that simulated microgravity could induce changes in cardiac repolarization and antonomic nervous system (ANS) thus increasing arrhythmias susceptibility and being manifested by an increase in TWA. Accordingly, our aim was to evaluate changes in TWA, as well as the role of sympathetic drive, during head-up tilt-test (TT) before and after 5 days of HDBR.

\section{MATERIALS AND METHODS}

\section{A. Data acquisition and preprocessing}

Twenty-two male healthy subjects (age range 21-43 years) were recruited in the context of the European Space Agency

This work was supported by CIBER-BBN through Instituto de Salud Carlos III and FEDER (Spain), project TEC2010-21703-C03-02 funded by MINECO and FEDER, and by DGA and European Social Fund (EU) through Grupo Consolidado GTC (T30). This research has been performed thanks to the contribution of the Italian Space Agency (contract n. I/047/10/0, recipient Dr. EG Caiani). We are extremely grateful to all the personnel of ESA, MEDES and DLR involved in the bed rest studies for the support to the realization of our experiment, as well as to the experimental subjects for their dedicated collaboration.

A. Martín-Yebra, A. Pellegrini and E.G. Caiani are with Dipartimento di Elettronica, Informazione e Bioingegneria, Politecnico di Milano, Italy (*corresponding author e-mail: albapilar.martin@polimi.it).

A. Martín-Yebra, V. Monasterio, P. Laguna and J.P. Martínez are with Instituto de Investigación en Ingeniería de Aragón, Universidad de Zaragoza and CIBER-BBN, Zaragoza, Spain.
(ESA) bedrest studies. The experiment included 5 days of pre-bed-rest hospitalization (PRE), 5 days of HDBR and 5 days of post-bed-rest recovery (POST).

ECG signals considered in this study were acquired using a high-resolution $\left(f_{s}=1000 \mathrm{~Hz}\right)$ 12-lead 24-hours Holter digital recorder (H12+, Mortara Instrument Inc., Milwaukee, WI) during TT, before and at the end of HDBR. TT consisted of $5 \mathrm{~min}$ in supine position, followed by head-up tilt $\left(80^{\circ}, 30\right.$ min) with additional lower body negative pressure applied after. Stop criteria were defined as very low BP, extreme tachycardia or clinical symptoms.

Preprocessing of ECG recordings included QRS detection using a wavelet-based ECG delineator [4]. Baseline wander was removed in each lead with a cubic spline interpolation technique. The frequency content of TWA is located below $15 \mathrm{~Hz}$, so the ECG signal was low-pass filtered $\left(f_{c}=15 \mathrm{~Hz}\right)$ and downsampled to remove offband noise.

\section{B. TWA analysis}

Automatic TWA analysis was performed using a multilead scheme based on Periodic Component Analysis ( $\pi \mathrm{CA}$ ) to find the optimal linear transformation from the 8 standard leads (V1-V6, I and II) to a new set of leads (T1 ...T8) where the 2-beat periodicity of the ST-T complex was maximized. TWA, if present, is mostly projected into the first lead T1. Laplacian Likelihood Ratio (LLR) method for TWA estimation is then applied to the combined lead [5].

ECG records were analyzed in segments of 32 beats with a $50 \%$ overlap. The TWA amplitude in each segment $\left(V_{k}\right)$ was defined as the absolute value of the mean of the estimated TWA waveform, computed as the median difference between ST-T complexes of even and odd beats. Moreover, a stability criterion in HR was also imposed and only suitable segments were selected for automatic analysis. Three average TWA amplitude indices were computed: 1) $\mathbf{T} \mathbf{W A} \mathbf{A}_{\text {bas }}$ : during the 5 minutes preceding the TT (baseline); 2) $\mathbf{T W A}_{\text {tilt }}$ : during the first 4 minutes of TT; 3) TWA rec: during the first 5 minutes once supine position was restored.

\section{Heart rate variability}

Heart rate (HR) signal, derived from QRS detection marks, was corrected by integral pulse frequency modulation model [6] and sampled at $4 \mathrm{~Hz}$, obtaining the new signal $d_{\mathrm{HR}}(n)$. Heart rate variavility (HRV) was defined as $d_{\mathrm{HRV}}(n)=$ $d_{\mathrm{HR}}(n)-d_{\mathrm{HRM}}(n)$, where $d_{\mathrm{HRM}}(n)$ is the mean heart rate estimated by low-pass filtering $d_{\mathrm{HR}}(n)$ with a cut-off frequency of $0.03 \mathrm{~Hz}$. Three segments were considered to avoid HR transitions: 1) BAS, from minute 5 up to $30 \mathrm{sec}$ before the tilt; 2) TILT, from $30 \mathrm{sec}$ after TT starts to $4.5 \mathrm{~min}$; 3) REC, from $30 \mathrm{sec}$ after supine position was restored to 
TABLE I TWA AND HRV INDICES COMPUTED BEFORE (PRE) AND AFTER 5 DAYS OF HDBR (POST). RESULTS ARE EXPRESSED AS MEDIAN (INTER-QUARTILE RANGE)

\begin{tabular}{|c|c|c|}
\hline & PRE-HDBR & POST-HDBR \\
\hline $\mathbf{H R}_{\text {bas }}$ (beats/min) & $61.1(18.9)$ & $67.9(21.4)^{\dagger}$ \\
\hline $\mathbf{T W A}_{\mathbf{b a s}}(\mu \mathrm{V})$ & $7.60(6.87)$ & $5.45(4.95)$ \\
\hline $\mathbf{L F}_{\text {bas }}\left(s^{2}\right)$ & $0.0012(0.0006)$ & $0.0008(0.0006)^{\dagger}$ \\
\hline $\mathbf{H F}_{\text {bas }}\left(s^{2}\right)$ & $0.00049(0.00047)$ & $0.00025(0.00012)^{\dagger}$ \\
\hline $\mathbf{L F n}_{\text {bas }}$ (n.u) & $0.714(0.195)$ & $0.786(0.139)^{\dagger}$ \\
\hline $\mathbf{L F} / \mathbf{H F}_{\text {bas }}$ (n.u) & $2.49(2.67)$ & $3.66(2.72){ }^{\dagger}$ \\
\hline $\mathbf{H R}_{\text {tilt }}$ (beats/min) & $85.1(14.8)$ & $98.5(21.6)^{\dagger}$ \\
\hline $\mathbf{T W A}_{\text {tilt }}(\mu \mathrm{V})$ & $5.60(4.55)$ & $3.20(3.89)$ \\
\hline $\mathbf{L F}_{\text {tilt }}\left(s^{2}\right)$ & $0.0018(0.0012)$ & $0.0008(0.0011)^{\dagger}$ \\
\hline $\mathbf{H F}_{\text {tilt }}\left(s^{2}\right)$ & $0.00026(0.0003)$ & $0.00012(0.00015)^{\dagger}$ \\
\hline $\mathbf{L F n}_{\text {tilt }}$ (n.u) & $0.863(0.122)$ & $0.875(0.075)$ \\
\hline $\mathbf{L F} / \mathbf{H F}_{\text {tilt }}$ (n.u) & $6.32(9.23)$ & $7.03(6.44)$ \\
\hline $\mathbf{H R}_{\text {rec }}$ (beats/min) & $76.6(14.4)$ & $78.1(19.0)$ \\
\hline TWA $_{\text {rec }}(\mu \mathrm{V})$ & $6.08(7.68)$ & $6.78(4.92)$ \\
\hline $\mathbf{L F}_{\text {rec }}\left(s^{2}\right)$ & $0.0015(0.0016)$ & $0.0015(0.0010)$ \\
\hline $\mathbf{H F}_{\text {rec }}\left(s^{2}\right)$ & $0.00056(0.0010)$ & $0.00031(0.00036)^{\dagger}$ \\
\hline $\mathbf{L F n}_{\text {rec }}$ (n.u) & $0.745(0.188)$ & $0.780(0.113)^{\dagger}$ \\
\hline $\mathbf{L F} / \mathbf{H F}_{\text {rec }}$ (n.u) & $2.92(2.47)$ & $3.52(2.37)^{\dagger}$ \\
\hline
\end{tabular}

minute 5 of recovery. For each segment, the power spectrum of $d_{\mathrm{HRV}}(n)$ was computed by using the periodogram estimator. The power in the LF $(0.04$ to $0.15 \mathrm{~Hz})$ and HF $(0.15$ to 0.4 $\mathrm{Hz}$ ) bands, $P_{\mathrm{LF}}$ and $P_{\mathrm{HF}}$, was estimated by integrating the power spectrum in the corresponding bands. Then, $P_{\mathrm{LF}}$ was expressed also in normalized units $\left(P_{\mathrm{LFn}}\right)$ and the ratio of sympatho-vagal absolute power $\left(R_{\mathrm{LF} / \mathrm{HF}}\right)$ was computed.

\section{RESULTS}

In Table I, results of HR, TWA indices and HRV analysis are presented. An increase in HR was found at BAS and TILT when comparing PRE vs POST. Despite this increment, and although it is known that TWA is a HR-dependent phenomenon, TWA indices were unchanged. As regards sympathetic activity, during BAS and REC, an increment in $P_{\mathrm{LFn}}$ and $R_{\mathrm{LF} / \mathrm{HF}}$ was found, evidencing a shift in the ANS balance at POST in these two phases but not during TILT.

Significant differences between PRE and POST in orthostatic tolerance times (OTT) were found (median (IQR): 38.7 (6.5) vs 8.3 (26.8) min, Wilcoxon signed-rank test, $p<0.001)$, but with no correlation with TWA indices $\left(\mathbf{T W A}_{\text {tilt }}\right.$ and TWA $_{\text {rec }}$ ).

Based on OTT at POST, we subdivided the subjects into two groups: 1) GShort, with OTT $\leq 30 \mathrm{~min}$; 2) GLong, with OTT $\geq 30 \mathrm{~min}$. Three subjects were excluded as they presented short OTT $(7.9,22$ and $24.3 \mathrm{~min})$ already at PRE and even shorter OTT $(3.5,3.9,8.3 \mathrm{~min}$ respectively) at post. Thus, 12 subjects were included in the GShort group, whereas the remaining 7 were included in GLong.

When comparing these groups, TWA $\mathbf{A}_{\text {tilt }}$ at POST was higher in GShort than in GLong (4.6 (7.02) vs. 1.92 (2.12) $\mu \mathrm{V}$, Mann-Whitney test, $p=0.028)$, which may suggests that subjects who were more prone to show higher orthostatic intolerance to tilt induced by HDBR presented a potentially higher cardiovascular risk in terms of TWA (or higher electrical instability in the repolarization phase). No other differences were found in terms of TWA, while at PRE during TILT $P_{\mathrm{LFn}}$ and $R_{\mathrm{LF} / \mathrm{HF}}$ both increased compared to BAS in GShort $(0.70(0.18)$ vs $0.90(0.10)$ n.u. and 2.34 (2.67) vs 9.68 (11.54) n.u, Wilcoxon signed-rank test, $p=0.002$, respectively) but not in GLong (0.77 (0.21) vs $0.78(0.16)$ n.u. and 3.38 (2.63) vs 3.62 (5.46) respectively), suggesting that sympathetic drive during TT increased in particular in GShort. After HDBR, ANS response to tilt was still evident in both groups, but without differences among them.

\section{DISCUSSION AND CONCLUSION}

We studied the potential changes in ventricular repolarization induced by HDBR by means of TWA and its possible relationship with ANS activity under TT orthostatic stress conditions. To our knowledge , this is the first study that attempts a description of TWA phenomenon during tilt test.

A significant shortening in OTT after HDBR was found, indicative of initial alterations in ANS activity after simulated microgravity. Based on these OTT changes and on the proposed subject classification, subjects classified as GShort presented at PRE a significant increase in both $P_{\mathrm{LFn}}$ and $R_{\mathrm{LF} / \mathrm{HF}}$ in contrast to the GLong group. These results are in agreement with previous studies in which in syncopal and postural orthostatic tachycardia syndrome patients, the reaction of sympathetic tone to orthostasis was more severe compared to control healthy subjects [7]. In addition, higher TWA $_{\text {tilt }}$ values were found in this group, suggesting higher electrical instability induced by HDBR associated to cardiac deconditioning.

Despite these differences, and the expected fact that higher TWA values are associated to higher sympathetic activation TWA $_{\text {tilt }}$ was found lower than $\mathbf{T W A} \mathbf{A}_{\text {bas }}$, while $P_{\mathrm{LFn}}$ and $R_{\mathrm{LF} / \mathrm{HF}}$ always increased with tilt. Nonetheless, the relationship between both phenomenona remains controversial [8], [9], and further research would be needed to further elucidate it.

In conclusion, 5 days are not long enough to produce significant changes in TWA parameters, reflecting increased heterogeneity in ventricular repolarization. Nevertheless, higher TWA values during tilt were observed in subjects with shorter OTT, which may be an indication of initial changes in the myocardial substrate.

\section{REFERENCES}

[1] Charles JB JB, Bungo MW, Fortner W. "Cardiopulmonary function. Space. Physiology and Medicine", 3rd Edition 1994; 286-304

[2] Hawkins WR, Zieglschmid JF. "Clinical aspects in crew health. Biomedical results of Apollo 1975"; (NASA SP-386) 286-304

[3] Verrier RL et al, "Microvolt T-wave alternans physiological basis, methods of measurement, and clinical utility - consensus guideline by International Society for Holter and Noninvasive Electrocardiology". J Am Coll Cardiol 2011; 58(13):1309-24

[4] Martínez JP, Almeida R, Olmos S, Rocha AP, Laguna P. "Waveletbased ECG delineator: evaluation on standard databases". IEEE Trans Biomed Eng 2004;51:570-581

[5] Monasterio V, Clifford GD, Laguna P, Martínez JP. "A multilead scheme based on periodic component analysis for T-wave alternans analysis in the ECG". Ann Biomed Eng 2010;38(8):2532-2541

[6] Mateo J, Laguna P. "Analysis of heart rate variability in the presence of ectopic beats using the heart timing signal". IEEE Trans Biomed Eng 2003;50:334-343

[7] Duplyakov D, Golovina G, Sysuenkova E, Garkina S. "Can the result of a tilt test be predicted in the first five minutes?" Cardiology Journal. 2011;18(5):521-6

[8] Schwartz PJ, Malliani A. "Electrical alternation of the T-wave: clinical and experimental evidence of its relationship with the sympathetic nervous system and with the long QT syndrome". Am Heart J 1975;89:45-50

[9] Kaufman ES, Mackall JA, Julka B, Drabek C, Rosenbaum, DS. "Influence of heart rate and sympathetic stimulation on arrhythmogenic TWA". Am J Physiol Heart Circ Physiol 2000; 279: 1248-1255 